\title{
Inverse Problems for Difference Equations with Quadratic Eigenparameter Dependent Boundary Conditions-II
}

\author{
Sonja Currie, Anne Love \\ School of Mathematics, University of the Witwatersrand, Johannesburg, South Africa \\ Email:Sonja.Currie@wits.ac.za,Anne.Love@wits.ac.za
}

How to cite this paper: Currie, S. and Love, A. (2016) Inverse Problems for Difference Equations with Quadratic Eigenparameter Dependent Boundary Conditions-II. Advances in Pure Mathematics, 6, 625-632. http://dx.doi.org/10.4236/apm.2016.610051

Received: April 29, 2016

Accepted: September 9, 2016

Published: September 12, 2016

Copyright $\odot 2016$ by authors and Scientific Research Publishing Inc. This work is licensed under the Creative Commons Attribution International License (CC BY 4.0).

http://creativecommons.org/licenses/by/4.0/ (c) (i) Open Access

\begin{abstract}
The following inverse problem is solved-given the eigenvalues and the potential $b(n)$ for a difference boundary value problem with quadratic dependence on the eigenparameter, $\lambda$, the weights $c(n)$ can be uniquely reconstructed. The investigation is inductive on $m$ where $m+1$ represents the number of unit intervals and the results obtained depend on the specific form of the given boundary conditions. This paper is a sequel to [1] which provided an algorithm for the solution of an analogous inverse problem, where the eigenvalues and weights were given and the potential was uniquely reconstructed. Since the inverse problem considered in this paper contains more unknowns than the inverse problem considered in [1], an additional spectrum is required more often than was the case in [1].
\end{abstract}

\section{Keywords}

Difference Equations, Inverse Problem, Boundary Value Problems, Spectrum, Eigenvalues

\section{Introduction}

Inverse problems in general are important in modern-day mathematics as they appear in many situations in physics, engineering, biology and medicine. This paper deals with inverse problems pertaining to a special type of second order difference equation. A comprehensive introduction to difference equations can be found, for example, in [2] and [3], amongst others. In particular, inverse problems for Sturm-Liouville difference equations with Dirichlet boundary conditions have been considered recently by Bohner and Koyunbakan in [4] where they show that the specification of the eigenvalues and 
weights uniquely determines the potential. In addition, they also prove that if the potential is symmetric, then it is uniquely determined by the eigenvalues only-this result can also be found in [5] where it is proved using different methods.

This paper is a sequel to [1] where the following second order difference equation was considered

$$
c(n) y(n+1)-b(n) y(n)+c(n-1) y(n-1)=-c(n) \lambda y(n),
$$

where $c(n)>0$ represents the weights associated with a potential function, $b(n)$. The boundary conditions, which were imposed respectively at the initial and terminal endpoints, had the general form

$$
\begin{gathered}
y(-1)=\left[a \lambda+b-\sum_{k=1}^{s} \frac{c_{k}}{\lambda-d_{k}}\right] y(0):=\operatorname{Ay}(0), \quad a \geq 0, c_{k}>0, \\
y(m-1)=\left[\alpha \lambda+\beta-\sum_{j=1}^{p} \frac{\gamma_{j}}{\lambda-\delta_{j}}\right] y(m):=\Lambda y(m), \quad \alpha \leq 0, \gamma_{j}<0 .
\end{gathered}
$$

Given the weights and the eigenvalues for the above boundary value problem with $s \leq 1$ and $p \leq 1$, a unique reconstruction of the potential was obtained, see [1] for details. This can be considered as a generalization of the results obtained in [4] in that more general boundary conditions are considered.

We now investigate the following inverse problem. Given a spectrum for a boundary value problem of the form (1), (2) and (3), with $s \leq 1$ and $p \leq 1$, together with the potentials $b(n)$, we prove that provided the number of eigenvalues exceeds $m+1$, where $m+1$ is the number of unit intervals, it is possible to uniquely reconstruct the weights $c(n)$. If the number of eigenvalues is less than $m+1$ we will require a second spectrum corresponding to a boundary value problem with exactly the same equation and boundary conditions of the same form in order to obtain a unique solution for $c(n)$.

The paper has the following structure. The proof of the above inverse problem is done inductively beginning with the cases of $m=1,2 \quad(s \leq 1$ and $p \leq 1)$ which is considered in Section 2. The inductive process continues in Section 3 with the cases corresponding to $m=3$. The main result of this paper is a generalization of the results obtained in Sections 2 and 3 and is given in Section 4, in particular Theorem 4.1.

As we do not have experimental data for the two examples presented in Section 3, the eigenvalues used are obtained by first solving the "forward" problem. Consequently, the theoretical results for certain of the inverse problems are then verified using these eigenvalues.

An important result concerning the number of eigenvalues associated with a particular boundary value problem was proved in [6] and will be used throughout this paper.

Theorem 1.1. Consider the boundary value problem given by Equation (1) for $n=0, \cdots, r-1$ together with boundary conditions (2) and (3) where $m=r$. This problem has

1) $s+p+r+1$ eigenvalues if $\alpha<0$,

2) $s+p+r$ eigenvalues if $\alpha=0$ and $\beta \neq 0$,

3) $s+p+r-1$ eigenvalues if $\alpha=\beta=0$. 
(Note that the number of unit intervals considered is $r+1$ ).

\section{Results for $m=1,2$}

In this section we investigate how to reconstruct the difference boundary value problem using a given spectrum or spectra. That is, how does the given spectrum/spectra, together with the potential function $b(0), b(1)$ provide us with a method of computing the associated weights $c(n), n=-1,0,1$.

Consider (1) with boundary conditions

$$
\begin{gathered}
y(-1)=\left[a \lambda+b-\frac{c}{\lambda-d}\right] y(0):=A y(0), \quad a \geq 0, c \geq 0, \\
y(m-1)=\left[\alpha \lambda+\beta-\frac{\gamma}{\lambda-\delta}\right] y(m):=\Lambda y(m), \quad \alpha \leq 0, \gamma \leq 0 .
\end{gathered}
$$

The cases of $c=0$ or $\gamma=0$ correspond respectively to $s=0$ or $p=0$ in (2) and (3).

In certain instances it is necessary to consider a second boundary value problem in order to obtain unique results. The second problem will be given by (1) with boundary conditions of the form

$$
\begin{gathered}
y(-1)=\left[\tilde{a} \mu+\tilde{b}-\frac{\tilde{c}}{\mu-\tilde{d}}\right] y(0):=\tilde{A} y(0), \quad \tilde{a} \geq 0, \tilde{c} \geq 0, \\
y(m-1)=\left[\tilde{\alpha} \mu+\tilde{\beta}-\frac{\tilde{\gamma}}{\mu-\tilde{\delta}}\right] y(m):=\tilde{\Lambda} y(m), \quad \tilde{\alpha} \leq 0, \tilde{\gamma} \leq 0 .
\end{gathered}
$$

The case $m=1$ is a "special" case in that if there is only one eigenvalue then two spectra are required in order to reconstruct $c(-1), c(0)$ uniquely. If there are two or more eigenvalues, then only one spectrum is needed in order to obtain a unique solution for $c(-1)$ and $c(0)$. The results for $m=1$ can be split into four cases (according to the number of given eigenvalues) and these mirror the results obtained in [1] except that the values of $b(0)$ are known and $c(-1), c(0)$ are uniquely reconstructed.

The cases for $m=2$ follow along the lines of those in [1] if the weights and potentials are interchanged and with the proviso that if the number of eigenvalues is greater than or equal to 3 then only one spectrum is required but if the number of eigenvalues is less than 3 then two spectra are necessary to produce unique results. In this case, we are given $b(0)$ and $b(1)$ and can uniquely reconstruct $c(-1), c(0)$ and $c(1)$.

\section{Main Results for $m=3$}

As the inequalities for $m=1,2$ differ from one another, we now extend these results to include $m=3$. There is an increased number of variables that need to be reconstructed which increases the technicality of the proofs. That is, given the spectrum/spectra and the potentials $b(0), b(1), b(2)$ can we uniquely reconstruct the weights $c(n)$, $n=-1,0,1,2$ ?

Again, we will split the inverse problem for $m=3$ into four cases depending on the 
coefficients in the boundary conditions (4), (5) and/or (6), (7). We begin with the cases where we require two spectra to reconstruct the weights uniquely.

Theorem 3.1. Consider the boundary value problems (1), (4), (5) and (1), (6), (7) where

1) $\alpha=\beta=0, c=0, \gamma \neq 0$ and $\tilde{\alpha}=\tilde{\beta}=0, \tilde{c}=0, \tilde{\gamma} \neq 0$;

2) $\alpha=0, \beta \neq 0, c=\gamma=0$ and $\tilde{\alpha}=0, \tilde{\beta} \neq 0, \tilde{c}=\tilde{\gamma}=0$.

The boundary value problems corresponding to 1) and 2) have three eigenvalues, say $\lambda_{0}, \lambda_{1}, \lambda_{2}$ for (1), (4), (5) and $\mu_{0}, \mu_{1}, \mu_{2}$ for (1), (6), (7). Given $\lambda_{0}, \lambda_{1}, \lambda_{2}, \mu_{0}$, $\mu_{1}, \mu_{2}$ together with $b(0), b(1)$ and $b(2)$, the weights $c(-1), c(0), c(1)$ and c(2) may be uniquely reconstructed in either case.

Proof. From Theorem 1.1, for both cases 1) and 2), it is clear that the boundary value problems each have three eigenvalues.

1) Assume $\alpha=\beta=0, c=0, \gamma \neq 0$ and $\tilde{\alpha}=\tilde{\beta}=0, \tilde{c}=0, \tilde{\gamma} \neq 0$ in (4), (5) and (6), (7). Then at $n=0,(1)$ together with (4) gives

$$
\frac{b(0)}{c(0)}-(a \lambda+b) \frac{c(-1)}{c(0)}-\lambda=\frac{y(1)}{y(0)}:=A_{1} \text {. }
$$

Next, evaluating (1) at $n=1$ and using (8) we obtain

$$
\frac{b(1)}{c(1)}-\frac{1}{A_{1}} \frac{c(0)}{c(1)}-\lambda=\frac{y(2)}{y(1)}:=A_{2} .
$$

Also, at $n=2$ we have

$$
\frac{b(2)}{c(2)}-\frac{1}{A_{2}} \frac{c(1)}{c(2)}-\lambda=\frac{y(3)}{y(2)}:=A_{3} .
$$

Then applying (5) gives the equation

$$
\frac{b(2)}{c(2)}-\frac{1}{A_{2}} \frac{c(1)}{c(2)}-\lambda=\frac{\lambda-\delta}{\gamma}
$$

which on simplification yields a polynomial in $\lambda$ of degree three namely,

$$
\begin{aligned}
0= & {\left[b(0) b(1) b(2) \gamma-b(2) c(0)^{2} \gamma-b(0) c(1)^{2} \gamma-b b(1) b(2) c(-1) \gamma\right.} \\
& \left.+b c(-1) c(1)^{2} \gamma+b(0) b(1) c(2) \delta-c(0)^{2} c(2) \delta-b b(1) c(2) c(-1) \delta\right] \\
& +\lambda\left[-b(0) b(1) c(2)+c(0)^{2} c(2)+b b(1) c(2) c(-1)-b(1) b(2) c(0) \gamma\right. \\
& -b(0) b(2) c(1) \gamma+c(0) c(1)^{2} \gamma-b(0) b(1) c(2) \gamma+c(0)^{2} c(2) \gamma-a b(1) b(2) c(-1) \gamma \\
& +b b(2) c(1) c(-1) \gamma+a c(1)^{2} c(-1) \gamma+b b(1) c(2) c(-1) \gamma-b(1) c(0) c(2) \delta \\
& -a b(1) c(2) c(-1) \delta-b(0) c(1) c(2) \delta+b c(1) c(2) c(-1) \delta] \\
& +\lambda^{2}[b(1) c(0) c(2)+b(0) c(1) c(2)+a b(1) c(2) c(-1)-b c(1) c(2) c(-1) \\
& +b(2) c(0) c(1) \gamma+b(1) c(0) c(2) \gamma+b(0) c(1) c(2) \gamma+a b(2) c(1) c(-1) \gamma \\
& +a b(1) c(2) c(-1) \gamma-b c(1) c(2) c(-1) \gamma+c(0) c(1) c(2) \delta+a c(1) c(2) c(-1) \delta] \\
& +\lambda^{3}[-c(0) c(1) c(2)-a c(1) c(2) c(-1)-c(0) c(1) c(2) \gamma-a c(1) c(2) c(-1) \gamma] .
\end{aligned}
$$

This can be rewritten in the form 


$$
\lambda^{3}+\mathbb{A} \lambda^{2}+\mathbb{B} \lambda+\mathbb{C}=0
$$

The eigencondition is given by

$$
\lambda^{3}+\left(-\lambda_{0}-\lambda_{1}-\lambda_{2}\right) \lambda^{2}+\left(\lambda_{0} \lambda_{1}+\lambda_{0} \lambda_{2}+\lambda_{1} \lambda_{2}\right) \lambda+\left(-\lambda_{0} \lambda_{1} \lambda_{2}\right)=0
$$

For (1), (6) and (7) we obtain the same third order equation as (12) with $\lambda$ replaced by $\mu$ and $a, b, \gamma, \delta$ replaced by their tilde $(\sim)$ versions.

In addition, the eigencondition in this case is given by

$$
\mu^{3}+\left(-\mu_{0}-\mu_{1}-\mu_{2}\right) \mu^{2}+\left(\mu_{0} \mu_{1}+\mu_{0} \mu_{2}+\mu_{1} \mu_{2}\right) \mu+\left(-\mu_{0} \mu_{1} \mu_{2}\right)=0 .
$$

Equating coefficients of $\lambda^{2}, \lambda^{1}, \lambda^{0}$ in (12) and (13) results in

$$
\mathbb{A}=-\lambda_{0}-\lambda_{1}-\lambda_{2}, \quad \mathbb{B}=\lambda_{0} \lambda_{1}+\lambda_{0} \lambda_{2}+\lambda_{1} \lambda_{2} \text { and } \mathbb{C}=-\lambda_{0} \lambda_{1} \lambda_{2},
$$

and similarly considering the coefficients of $\mu^{2}, \mu^{1}$ and $\mu^{0}$ yields

$$
\tilde{\mathbb{A}}=-\mu_{0}-\mu_{1}-\mu_{2}, \quad \tilde{\mathbb{B}}=\mu_{0} \mu_{1}+\mu_{0} \mu_{2}+\mu_{1} \mu_{2} \text { and } \tilde{\mathbb{C}}=-\mu_{0} \mu_{1} \mu_{2} .
$$

Solving the six simultaneous equations gives $c(-1), c(0), c(1)$ and $c(2)$ uniquely.

2) Suppose that $\alpha=0, \beta \neq 0, c=\gamma=0$ and $\tilde{\alpha}=0, \tilde{\beta} \neq 0, \tilde{c}=\tilde{\gamma}=0$. Then (8), (9) and (10) hold and (11) becomes

$$
\frac{b(2)}{c(2)}-\frac{1}{A_{2}} \frac{c(1)}{c(2)}-\lambda=\frac{1}{\beta}
$$

This can be rewritten as a cubic polynomial i.e. in the form (12) where $\mathbb{A}, \mathbb{B}$ and $\mathbb{C}$ can be found using Mathematica or similar programmes.

Again for (1), (6) and (7) we obtain the same cubic polynomial as above with $\lambda$ replaced by $\mu$ and $a, b, \gamma, \delta$ replaced by their tilde $(\sim)$ versions. The eigenconditions are given by (13) and (14) thus, Equations (15) and (16) hold. Hence, we can again solve the six simultaneous equations uniquely for $c(-1), c(0), c(1)$ and $c(2)$.

Theorem 3.2. Consider the boundary value problem (1), (4) and (5) where

1) $\alpha=0, \quad \beta \neq 0, c \neq 0, \gamma=0$ and $\tilde{\alpha}=0, \tilde{\beta} \neq 0, c \neq 0, \tilde{\gamma}=0$;

2) $\alpha=0, \beta \neq 0, c=0, \gamma \neq 0$ and $\tilde{\alpha}=0, \tilde{\beta} \neq 0, c=0, \tilde{\gamma} \neq 0$;

3) $\alpha=\beta=0, c \neq 0, \quad \gamma \neq 0$ and $\tilde{\alpha}=\tilde{\beta}=0, \quad c \neq 0, \tilde{\gamma} \neq 0$;

4) $\alpha<0, c=\gamma=0$ and $\tilde{\alpha}<0, c=\tilde{\gamma}=0$.

The boundary value problem corresponding to any of the four cases above has four eigenvalues, say $\lambda_{0}, \lambda_{1}, \lambda_{2}$ and $\lambda_{3}$ for (1), (4), (5) and $\mu_{0}, \mu_{1}, \mu_{2}$ and $\mu_{3}$ for (1), (6), (7). The weights $c(-1), c(0), c(1)$ and $c(2)$ may be uniquely reconstructed in any of these cases assuming we are given the eigenvalues together with $b(0), b(1)$ and $b(2)$.

Proof. This uses the procedure outlined in Theorem 3.1 above. It is similar to the proof of Theorem 3.2 in [1] but with increased dependence on $\lambda$ as a polynomial of degree four is obtained. The eigencondition is also a fourth order polynomial namely,

$$
\begin{aligned}
0= & \lambda^{4}+\left(-\lambda_{0}-\lambda_{1}-\lambda_{2}-\lambda_{3}\right) \lambda^{3}+\left(\lambda_{0} \lambda_{1}+\lambda_{0} \lambda_{2}+\lambda_{0} \lambda_{3}+\lambda_{1} \lambda_{2}+\lambda_{1} \lambda_{3}+\lambda_{2} \lambda_{3}\right) \lambda^{2} \\
& +\left(-\lambda_{0} \lambda_{1} \lambda_{2}-\lambda_{0} \lambda_{1} \lambda_{3}-\lambda_{0} \lambda_{2} \lambda_{3}-\lambda_{1} \lambda_{2} \lambda_{3}\right) \lambda+\left(\lambda_{0} \lambda_{1} \lambda_{2} \lambda_{3}\right),
\end{aligned}
$$


and similarly for $\mu$. Equating relevant coefficients of the powers of $\lambda$ and $\mu$ and solving the resulting equations simultaneously, yields unique values for $c(-1), c(0)$, $c(1)$ and $c(2)$.

Theorem 3.3. Consider the boundary value problem (1), (4) and (5) where

1) $\alpha=0, \beta \neq 0, c \neq 0, \gamma \neq 0$;

2) $\alpha<0, c \neq 0, \gamma=0$;

3) $\alpha<0, c=0, \gamma \neq 0$.

Given the five eigenvalues $\lambda_{i}, i=0,1,2,3$ associated with the boundary value problems above, as well as the values $b(0), b(1)$ and $b(2)$, it is possible to reconstruct $c(-1), c(0), c(1)$ and $c(2)$ uniquely.

Proof. In all three cases, starting with $n=0$ in (1) and imposing the boundary conditions where appropriate, yields a fifth order polynomial in $\lambda$, that is

$$
\lambda^{5}+\mathbb{A} \lambda^{4}+\mathbb{B} \lambda^{3}+\mathbb{C} \lambda^{2}+\mathbb{D} \lambda+\mathbb{E}=0 .
$$

The associated eigencondition is also a fifth order polynomial given by

$$
\lambda^{5}+\hat{A} \lambda^{4}+\hat{B} \lambda^{3}+\hat{C} \lambda^{2}+\hat{D} \lambda+\hat{E}=0,
$$

where

$$
\begin{aligned}
\hat{A}= & -\lambda_{0}-\lambda_{1}-\lambda_{2}-\lambda_{3}-\lambda_{4}, \\
\hat{B}= & \lambda_{0} \lambda_{1}+\lambda_{0} \lambda_{2}+\lambda_{1} \lambda_{2}+\lambda_{0} \lambda_{3}+\lambda_{1} \lambda_{3}+\lambda_{2} \lambda_{3}+\lambda_{3} \lambda_{4}+\lambda_{0} \lambda_{4}+\lambda_{2} \lambda_{4}+\lambda_{1} \lambda_{4}, \\
\hat{C}= & -\lambda_{0} \lambda_{1} \lambda_{2}-\lambda_{0} \lambda_{1} \lambda_{3}-\lambda_{0} \lambda_{2} \lambda_{3}-\lambda_{1} \lambda_{2} \lambda_{3}-\lambda_{0} \lambda_{1} \lambda_{4} \\
& -\lambda_{0} \lambda_{2} \lambda_{4}-\lambda_{1} \lambda_{2} \lambda_{4}-\lambda_{0} \lambda_{3} \lambda_{4}-\lambda_{1} \lambda_{3} \lambda_{4}-\lambda_{2} \lambda_{3} \lambda_{4}, \\
\hat{D}= & \lambda_{0} \lambda_{1} \lambda_{2} \lambda_{3}+\lambda_{0} \lambda_{1} \lambda_{2} \lambda_{4}+\lambda_{0} \lambda_{1} \lambda_{3} \lambda_{4}+\lambda_{0} \lambda_{2} \lambda_{3} \lambda_{4}+\lambda_{1} \lambda_{2} \lambda_{3} \lambda_{4}, \\
\hat{E}= & -\lambda_{0} \lambda_{1} \lambda_{2} \lambda_{3} \lambda_{4} .
\end{aligned}
$$

By equating relevant coefficients of powers of $\lambda$, unique solutions for $c(-1), c(0)$, $c(1)$ and $c(2)$ result from the simultaneous solution of the resulting equations.

Theorem 3.4. Assume that we have the boundary value problem (1), (4) and (5) with $\alpha<0, c \neq 0$ and $\gamma \neq 0$. Given the six eigenvalues $\lambda_{i}, i=0,1, \cdots, 5$ associated with this boundary value problem, together with the values $b(0), b(1)$ and $b(2)$, a unique reconstruction of $c(-1), c(0), c(1)$ and $c(2)$ is possible.

Proof. As per usual we start the evaluation of (1) at $n=0$ together with (4). Following procedures outlined above in (3.2) and [1], we obtain an equation which reduces to a sixth order polynomial in $\lambda$. Equating coefficients of this polynomial with those of the eigencondition provides a unique solution for $c(-1), c(0), c(1)$ and $c(2)$.

\section{Example 1}

To illustrate part (3) of Theorem 3.3, suppose that $\lambda_{0}=-2.67152, \lambda_{1}=1.56314$, $\lambda_{2}=-0.843923, \lambda_{4}=0.798491$ and $\lambda_{5}=0.0327864$ are the given eigenvalues corresponding to the boundary value problem

$$
\begin{gathered}
c(n) y(n+1)-n y(n)+c(n-1) y(n-1)=-\lambda c(n) y(n), \\
y(-1)=(3 \lambda-1) y(0),
\end{gathered}
$$




$$
y(2)=-2 \lambda+1-\frac{-4}{\lambda+2} y(3) .
$$

Clearly, it is seen that, $a=3, b=-1, c=0, \alpha=-2, \gamma=-4, \beta=1$. Using these values together with $b(0)=0, b(1)=1, b(2)=2$ and the method outlined in the theorems, one finds $\mathbb{A}, \mathbb{B}, \mathbb{C}, \mathbb{D}$ and $\mathbb{E}$ as given in (18). Equating these with the coefficients of (19) and simultaneously solving the five equations yields $c(-1)=1, c(0)=4$, $c(1)=9$ and $c(2)=16$.

\section{Example 2}

Assume that we are given eigenvalues $\lambda_{0}=-2.67152, \lambda_{1}=1.56314$, $\lambda_{2}=-0.843923, \lambda_{4}=0.798491$ and $\lambda_{5}=0.0327864$ corresponding to the boundary value problem

$$
\begin{gathered}
c(n) y(n+1)-(n+2)^{2} y(n)+c(n-1) y(n-1)=-\lambda c(n) y(n), \\
y(-1)=\left(1-\frac{4}{\lambda+1}\right) y(0), \\
y(2)=-\frac{-1}{\lambda+2} y(3) .
\end{gathered}
$$

To illustrate Theorem 3.2(3), suppose also that $\mu_{0}=3.43996, \mu_{1}=2.39846$, $\mu_{2}=1.40648$, and $\mu_{3}=-0.161577$ are the eigenvalues corresponding to the boundary value problem

$$
\begin{gathered}
c(n) y(n+1)-(n+2)^{2} y(n)+c(n-1) y(n-1)=-\lambda c(n) y(n), \\
y(-1)=\left(-\frac{2}{\lambda-1}\right) y(0), \\
y(1)=-\frac{-5}{\lambda+1} y(3) .
\end{gathered}
$$

Note that the two boundary value problems above are of the same form i.e. they have exactly the same equation and their boundary conditions are of the same type. In the first problem, $a=0, b=1, c=4, \alpha=\beta=0, \gamma=-1$ while $\tilde{a}=\tilde{b}=0, \tilde{c}=2, \tilde{\alpha}=\tilde{\beta}=0, \tilde{\gamma}=-5$ in the second problem and $b(0)=4, b(1)=9$, $b(2)=16$ for both problems. Using these values routine calculations give $\mathbb{A}, \mathbb{B}, \mathbb{C}$, $\mathbb{D}, \tilde{\mathbb{A}}, \tilde{\mathbb{B}}, \tilde{\mathbb{C}}$ and $\tilde{\mathbb{D}}$. Simultaneously solving the eight equations yields $c(-1)=2, c(0)=3, c(1)=4$ and $c(2)=5$.

\section{General Case $m=r$}

As mentioned previously, the case of $m=1$ is a special case in that if the number of eigenvalues is less than $r+1$, then two spectra are required. However, if the number of eigenvalues is greater than or equal to $r+1$ then only one spectrum is required. Using the previous two sections and with $m>1$, we inductively obtain results for the general case where $m=r$. That is, given the spectrum/spectra and the potentials $b(n)$, $n=0, \cdots, r-1$ the theorem below provides an algorithmic method enabling one to uniquely reconstruct the weights $c(n), n=-1, \cdots, r-1$.

Theorem 4.1. For $r>1$, assume that we are given the spectrum $\lambda_{0}, \cdots, \lambda_{k-1}$ of the 
boundary value problem (1) with boundary conditions (4) and (5) where the number of eigenvalues $k$ is calculated according to Theorem 1.1. In addition, suppose that the potentials $b(0), \cdots, b(r-1)$ are known,

1) if $k>r+1$ then the weights $c(-1), \cdots, c(r-1)$ can be uniquely reconstructed;

2) if $k \leq r+1$ and we are given a second spectrum $\mu_{0}, \cdots, \mu_{k-1}$ corresponding to the boundary value problem (1) with boundary conditions (6) and (7) then the weights $c(-1), \cdots, c(r-1)$ can be uniquely reconstructed.

Proof. Follows as in ([1], Theorem 4.1) where the $k$ simultaneous equations in 1$)$ and $2 k$ simultaneous equations in 2 ) are now solved to find unique values for $c(-1), \cdots, c(r-1)$.

Remark: 1) It is not possible for the number of eigenvalues of (1), (4) and (5) to be less than $m$ as this would imply a Dirichlet boundary condition at $m-1$, meaning that the interval shrinks and we wish to maintain the number of unit intervals as $m+1$.

2) It should be noted that because there are more weights than potentials, two spectra are required more often than in [1] in order to obtain a unique reconstruction of the weights.

\section{Acknowledgements}

We thank the Editor and the referee for their comments. Research of S. Currie is supported by NRF grant no. IFR2011040100017. This support is greatly appreciated.

\section{References}

[1] Currie, S. and Love, A. (Submitted) Inverse Problems for Difference Equations with Quadratic Eigenparameter Dependent Boundary Conditions.

[2] Elaydi, S. (2005) An Introduction to Difference Equations. Springer, Berlin.

[3] Kelley, W.G. and Peterson, A.C. (1991) Difference Equations: An Introduction with Applications. Academic Press, New York.

[4] Bohner, M. and Koyunbakan, H. (2016) Inverse problems for Sturm-Liouville Difference Equations. Filomat, 30, 1297-1304. http://dx.doi.org/10.2298/fil1605297b

[5] Hald, O.H. (1976) Discrete Inverse Sturm-Liouville Problems. I. Uniqueness for Symmetric Potentials. Numerische Mathematik, 27, 249-256. http://dx.doi.org/10.1007/BF01396645

[6] Currie, S. and Love, A. (2013) Hierarchies of Difference Boundary Value Problems Continued. Journal of Difference Equations and Applications, 19, 1807-1827. 
Submit or recommend next manuscript to SCIRP and we will provide best service for you:

Accepting pre-submission inquiries through Email, Facebook, LinkedIn, Twitter, etc. A wide selection of journals (inclusive of 9 subjects, more than 200 journals)

Providing 24-hour high-quality service

User-friendly online submission system

Fair and swift peer-review system

Efficient typesetting and proofreading procedure

Display of the result of downloads and visits, as well as the number of cited articles

Maximum dissemination of your research work

Submit your manuscript at: http://papersubmission.scirp.org/ 